Baldocchi, Russ

[15]

\section{Hybridization to oligonucleotide arrays for the assessment of gene copy number}

Russ Baldocchi ${ }^{1}$, Richard Glynne ${ }^{2}$, Dave Kowbel ${ }^{1}$, Ed Tom², Colin Collins ${ }^{1}$, David Mack ${ }^{2} \&$ Joe Gray ${ }^{1}$

${ }^{1}$ University of California San Francisco Cancer Center, San Francisco, California, USA

${ }^{2}$ Eos Biotechnology, Inc., South San Francisco, California, USA

Array-based comparative genomic hybridization (CGH) provides a higher-resolution and more quantitative alternative to chromosome CGH for the assessment of abnormalities in genomic copy number. In array-based CGH analyses published to date, array elements have been composed of cloned DNA sequences (such as P1s, BACs or complementary DNAs), and thus copy number abnormalities can be mapped from data on the genomic mapping of such clones. These advanced methods are limited by difficulties in the production of cloned DNAs and distortions in the data arising from elements in the arrays that are present at multiple copies in the normal genome. To overcome some of these limitations, we have produced a low-complexity probe based on the polymerase chain reaction (PCR). In oligonucleotide array CGH, probes are prepared by multiplex PCR amplification using 10-50 primer pairs. Two 50-base oligonucleotides that map between the primer pairs serve as array elements. Because the linearity of this method apparently depends on primer complexity during PCR, we substituted adapter sequences (which make up the $5^{\prime}$ end of our PCR primers). We compared the performances of T7 and R24 adapters in a 24-plex PCR with one another and with previously obtained copy number data on several chromosome 20 loci in MCF7 cells obtained from fluorescence in situ hybridization. We found that use of the T7 adapters improved the amplitude of test-to-reference ratios 2.3-fold, suggesting less-dampened ratios and increased linearity in our assessment of copy number by this method. We will demonstrate the large-scale application of oligonucleotide array CGH to ovarian and breast cancer samples.

Basilion, J.P.

\section{Magnetic resonance imaging of HSV-based in vivo transgene expression}

Dagmar Hoegemann ${ }^{1}$, Tomo Ichikawa², E.A. Chiocca², Ralph Weissleder ${ }^{1} \&$ James P. Basilion ${ }^{1,2}$

${ }^{1}$ Center for Molecular Imaging Research and ${ }^{2}$ Neurosurgical Service, Molecular Neuro-Oncology Laboratory, Massachusetts General Hospital, Harvard Medical School, Boston, Massachusetts, USA

Real-time imaging of gene expression in vivo at high spatial resolution would significantly aid our ability to study and understand transgene expression in live animals and potentially in a clinical gene therapy setting. Magnetic resonance (MR) imaging has recently achieved spectacular image resolution $\left(50 \mu \mathrm{m}^{3}\right.$ voxel resolution in vivo), making possible imaging at very high resolutions in small animals, during development and in clinical practice. Recently we have developed gene-targeted MR imaging contrast agents that allow us to use clinical MR imaging systems to monitor gene expression in vivo. We have demonstrated that expression of an altered form of the transferrin receptor (ETR) can be imaged in vivo using MR imaging and ETR-targeted imaging probes ${ }^{1}$. ETR expression could be used as a surrogate marker to monitor viral delivery and expression of other transgenes in real time, in vivo and potentially in a clinical setting. To demonstrate the feasibility of this approach we have constructed amplicons, based on the herpes simplex virus, that co-express the ETR and different therapeutic or marker genes under the control of different constitutive promoters. In vitro we have shown that these amplicons drive the expression of multiple inserted genes and that the expression of the ETR is correlated with the expression of other genes contained within the same amplicon. Using these amplicons in a mouse xenograft brain tumor model we have demonstrated non-invasive MR imaging of in vivo transferred gene products. The results demonstrate the feasibility and power of this new approach; identification of targets overexpressed in different disease states will expand the potential application of this technology as a noninvasive diagnostic tool.

1. Hoegemann, D. et al. Nature Med. 6, 351-355 (2000).

Barrett, Michael [17]

\section{Transcriptional analysis of tetraploid epithelial cells in Barrett's esophagus}

Michael Barrett ${ }^{1}$, David Pritchard ${ }^{2}$, Judy Anderson ${ }^{2}$, Brian Reid $^{1} \&$ Peter Rabinovitch ${ }^{1,2}$

${ }^{1}$ Fred Hutchinson Cancer Research Center, Seattle, Washington, USA

${ }^{2}$ University of Washington, Seattle, Washington, USA

Increased $4 \mathrm{~N}$ (G2/tetraploid) fractions in patients with premalignant Barrett's esophagus are associated with loss of p53 function and are highly predictive of progression to aneuploidy and esophageal adenocarcinoma. Studies in Barrett's esophagus and model systems have suggested that tetraploid cells are unstable intermediates in the development of aneuploidy and cancer. We developed primary epithelial cultures from biopsies of patients with early stages of Barrett's esophagus. Each of four cultures had p53 lesions, p16 lesions or both that were also present in vivo, and each culture contained $12-25 \% 4 \mathrm{~N}$ cells, of which approximately $50 \%$ were G1 tetraploid cells. To study the molecular phenotypes of these tetraploid cells, we sorted $2 \mathrm{~N}$ and $4 \mathrm{~N}$ cells from each culture viably using Hoechst 33342 labeling. We stored diploid cells while sorted $4 \mathrm{~N}$ fractions were recultured for two weeks, then resorted to purify tetraploid populations. Total RNA from each sorted sample was used to interrogate Affymetrix FL6800 arrays. No genes were consistently underexpressed in $4 \mathrm{~N}$ compared with $2 \mathrm{~N}$ cells, whereas 32 genes $(0.9 \%)$ showed consistently twofold increased expression in $4 \mathrm{~N}$ cells; 24 of 32 were named genes and all had functions associated with G2/M and related checkpoints. These included genes associated with centrosome regulation (nek-2, $p l K-1)$, the G2/M transition (CDC2, cyclin B, Wee1, CDC25) and mitosis (genes for topoisomerase II, mitosin, CENP-A, kinesin-related protein). In contrast there were no changes in the expression of a series of G1/S-specific genes, including RRM1, CDK4, PRAD1, CAD and MAPKKK5. Tetraploid BE G1 cells in Barrett's esophagus have dysregulated expression of $\mathrm{G} 2 / \mathrm{M}$ genes.

Baumbach-Reardon, Lisa

[18]

\section{Completed BRCA1/BRCA2 mutation analysis reveals a low rate of germline mutation in at-risk African American families}

Lisa Baumbach-Reardon ${ }^{1}$, Luis Gayol ${ }^{1}$, Tom Scholl2 ${ }^{2}$, Hugo Basterrechea ${ }^{1}$, Selina Smith ${ }^{1} \&$ J. Fernando Arena ${ }^{1}$

${ }^{1}$ University of Miami School of Medicine, Miami, Florida, USA

${ }^{2}$ Myriad Genetics, Inc., Salt Lake City, Utah, USA

The incidence of $B R C A 1$ germline mutations in at-risk individuals is controversial. In Caucasians the rate of detection of $B R C A 1$ mutations varies from $5 \%$ to $40 \%$. The incidence of $B R C A 1$ mutations and genetic variants in at-risk African Americans has 